\title{
Invasive evaluation of patients after reperfused STEMI: One-stop-shop for anatomy and physiology
}

\author{
Habib Samady, MD, FACC
}

\section{See related article, pp. 817-824}

Despite micro-remobilization and reperfusion injury associated with reperfusion therapy, the initial goal of therapy in patients with ST elevation myocardial infarction (STEMI) is to halt the ongoing ischemic insult to the myocardium by achieving myocardial reperfusion. Reperfusion can rarely occur spontaneously, and is facilitated by anti-platelet and anti-thrombotic therapy, but optimally requires percutaneous coronary intervention (PCI), fibrinolytic therapy, or combination pharmaco-invasive therapy. Patients who initially receive fibrinolytic but fail pharmacologic reperfusion are referred for emergent rescue PCI. Those patients who undergo successful primary or rescue PCI have the advantage of not only achieving myocardial reperfusion by recanalization of the obstructed vessel, but also revascularization of any residual epicardial stenosis such that any residual viable peri-infarct myocardium will not remain ischemic. However, as there are only a limited number of centers that can perform emergency mechanical reperfusion by primary PCI, the majority of STEMI patients who have access to contemporary medical therapy undergo pharmacologic reperfusion.

Once epicardial and microvascular reperfusion are achieved and the patient stabilized, the next priority is to identify residual hemodynamically significant lesions in the infarct and other myocardial beds that would require revascularization. Therefore, pharmacologically reperfused STEMI patients either undergo non-invasive testing or coronary angiography to identify residual

\footnotetext{
From the Division of Cardiology, Department of Medicine, Emory University School of Medicine, Atlanta, GA.

Dr. Samady has received research grants from Volcano Therapeutics. Reprint requests: Habib Samady, MD, FACC, Division of Cardiology, Department of Medicine, Emory University School of Medicine, Suite F606, 1365, Clifton Road, Atlanta, GA; hsamady@emory.edu. J Nucl Cardiol 2010;17:775-7.

$1071-3581 / \$ 34.00$

Copyright (c) 2010 American Society of Nuclear Cardiology.

doi:10.1007/s12350-010-9283-6
}

ischemia in the infarct bed and the remaining myocardium. Among patients who undergo non-invasive risk stratification, those with preserved ventricular function and no evidence of ischemia are managed medically and patients with reduced ventricular function and/or with inducible ischemia are referred for angiography. ${ }^{1}$

In the cardiac catheterization laboratory, in addition to anatomical assessment of lesion severity by angiography, physiologic evaluation of epicardial and microvascular beds can be performed using angioplastytype guide wires with pressure and velocity sensors. The epicardial lesion can be assessed by measuring fractional flow reserve (FFR) or hyperemic stenosis resistance (HSR), the microcirculation by measuring the index of microcirculatory resistance (IMR) or the hyperemic myocardial resistance index (HMR), and both epicardial and microcirculatory beds by measuring coronary flow reserve (CFR). ${ }^{2}$ So what tools in the cardiac catheterization laboratory are best suited to assess lesion severity in the post-infarct bed? Because FFR has been extensively validated in patient's normal ventricles and is now gaining traction with interventional cardiologists as a simple and robust physiologic index of lesion severity, ${ }^{3}$ if validated in this setting, it would be the ideal tool to assess lesion severity in reperfused STEMI patients.

Fractional flow reserve is defined as the ratio of the hyperemic flow distal to a stenosis to hyperemic normal flow. As flow is equal to pressure divided by resistance and resistance can be cancelled from both the numerator (stenotic flow) and the denominator (normal or aortic flow) when it is minimal and constant during hyperemia, then FFR can simply be calculated as the ratio of distal to aortic pressure during hyperemia. ${ }^{2}$ However, theoretically, when the microcirculatory resistance is elevated, such as after STEMI, pressure-derived FFR might underestimate the physiologic significance of a stenosis. In other words, the calculated FFR value would be higher in a post-infarct bed than for an identical lesion subtending a normal myocardial bed. However, proponents of FFR have argued that although microvascular resistance is elevated in the infarcted bed, it is nevertheless minimal and constant during hyperemia and therefore it can be cancelled from the numerator and the denominator without significant error in the FFR 
value. Furthermore, it has been argued that the FFR of a lesion subtending an infarct bed is higher than that of an identical lesion supplying a non-infarcted bed, the higher value accounts for the appropriate amount of residual viable myocardium in the infarcted bed. ${ }^{4}$

The complex pathophysiology of infarct healing provides several theoretical and practical challenges to the accurate identification of the hemodynamic severity of lesions in the infarcted bed. In the late post-infarction phase usually myocardial edema and stunning have resolved and infarct expansion and remodeling are complete, therefore, the major challenge in assessing the hemodynamic severity of a lesion supplying the infarct bed is increased myocardial resistance impairing the ability of the microcirculation to maximally vasodilate. This elevated microcirculatory resistance in the infarct bed can theoretically lead to underestimation of periinfarct ischemia caused by an epicardial stenosis in the infarct bed. To shed light on this debate, DeBruyne et $\mathrm{al}^{5}$ showed that FFR accurately identified inducible ischemia on single photon emission computerized tomography (SPECT) in patients $20 \pm 27$ days (range 6-570 days) after STEMI. Furthermore, he demonstrated that there was an inverse relationship between FFR and ventricular function after STEMI, such that FFR was lower in patients with more preserved myocardial function and hence viability.

The early peri-infarct period is particularly complex, where in addition to the microcirculatory infarction, there is microvascular stunning, thromboembolism with platelet and thrombus plugging, coronary vasospasm, endothelial dysfunction, and reperfusion injury. All these processes contribute to a dynamic and diminish microvascular reactivity, thereby potentially affecting both invasive and non-invasive physiologic testing. Indeed, myocardial flow reserve has been shown to be reduced early following infarction and improves over time. ${ }^{6}$ Thus, the fidelity of all vasodilator-dependent physiologic studies may be affected early after STEMI. The clinical implications of this phenomenon are important as many patients after reperfused infarction undergo stress testing or angiography with FFR determination of lesion severity. To evaluate the clinical utility FFR in the early post-infarct setting, we demonstrated that FFR of angiographically matched lesions subtending infarcted beds were almost identical to FFR of lesions subtending normal beds. ${ }^{7}$ In a subsequent study, we showed that FFR had excellent predictive value for identifying peri-infarct ischemia on SPECT and myocardial contrast echocardiography in patients 48-72 $\mathrm{h}$ after myocardial infarction. ${ }^{8}$ Despite these data supporting the value of FFR for detecting peri-infarct ischemia in the early post-infarct phase, outcome data are lacking using an FFR-guided revascularization strategy in patients with recently ruptured moderate plaque and therefore clinical judgment should be used to guide clinical decision making.

In this context the paper by Beleslin et $\mathrm{al}^{9}$ in this issue of the journal evaluated the relationship between baseline FFR and Nitroglycerine augmented rest SPECT in $26 \mathrm{pts}$ with prior STEMI to improvement in perfusion assessed by Gated SPECT 3 months after PCI. Importantly, PCI was performed $5 \pm 8$ months after STEMI. The authors found that baseline FFR was lower in patients with viability on SPECT (defined tracer uptake $\geq 55 \%$ ) compared to patients without SPECT viability, despite both the groups having similar angiographic lesion severity. They found FFR to correlate significantly with improvement in perfusion ( $>5 \%$ tracer uptake) after revascularization and baseline peak serum creatine phosphokinase levels. The authors conclude that lower values of FFR before angioplasty are associated with myocardial viability and functional improvement as assessed by SPECT perfusion scintigraphy. It should be noted that the findings of this study only apply to a limited patient population, i.e., stable patients with single vessel disease who are at least 7 days (mean of $5 \pm 8$ months) after stabilized STEMI. In the United States, invasive evaluation is usually performed within $48 \mathrm{~h}$ after STEMI, but in Europe and many other parts of the world patients often undergo angiography several months after their index STEMI. In addition, patients with multivessel disease, occluded infarct related arteries, previous PCI (either elective, primary, or rescue), or with unstable angina or heart failure at the time of their angiography were excluded. Other potential limitations of this study acknowledged by the authors include the use of single threshold definitions for viability assess ( $>55 \%$ Sestamibi uptake) and for improvement in perfusion $(>5 \%$ increase tracer uptake) and the lack of invasive microcirculatory testing such as IMR or HMR which would possibly unmask limitations in the diagnostic accuracy of FFR in this setting.

Despite its limited size and the aforementioned limitations, the study by Beleslin et al is the first in the literature to relate FFR to a surrogate of improved outcomes (improvement in perfusion on SPECT imaging) in the post-STEMI population and thus adds to the existing literature supporting the use of FFR to direct revascularization in this setting. Clearly, large randomized trials comparing non-invasive testing to combined angiography and FFR to guide revascularization in the reperfused STEMI population are warranted. Until the results of such studies are available, revascularization decisions in patients after stabilized myocardial infarction will likely continue to be individualized and may depend on the treating cardiologist's bias. In the interim 
with the advent of FFR, the use of the old adage referring to risk stratification of post-infarct patients "noninvasive testing provides physiologic information and invasive testing provides anatomic evaluation', can no longer be justified!

\section{Acknowledgment}

The author wishes to thank Dr. Michael C. McDaniel for his review of this manuscript.

\section{References}

1. Antman EM, Anbe DT, Armstrong PW, et al. ACC/AHA guidelines for the management of patients with ST-elevation myocardial infarction-executive summary. Circulation 2004;110:588-636.

2. Kern MJ, Samady H. Current concepts of integrated coronary physiology in the catheterization laboratory. J Am College Cardiol 2010;55:173-85.
3. Kern MJ, Lerman A, Bech JW, et al. Physiologic assessment of coronary artery disease in the cardiac catheterization laboratory: A scientific statement from the American Heart Association Committee on Diagnostic and Interventional Cardiac Catheterization, Council on Clinical Cardiology. Circulation 2006;114:1321-41.

4. Bishop A, Samady H. Fractional flow reserve: Critical review of an important adjunct to angiography. Am Heart J 2004;93(9):1102-6.

5. DeBruyne B, Pijls NHJ, Bartunek J, et al. Fractional flow reserve in patients with prior myocardial infarction. Circulation 2001;104: $157-62$.

6. Uren NG, Crake T, Lefroy DC, et al. Reduced coronary vasodilator function in infarcted and normal myocardium after myocardial infarction. N Engl J Med 1994;331:222-7.

7. McClish JC, Ragosta M, Powers ER, et al. Effect of acute myocardial infarction on the utility of fractional flow reserve for the physiologic assessment of the severity of coronary artery narrowing. Am J Cardiol 2004;93:1102-6.

8. Samady H, Lepper W, Powers ER, et al. Fractional flow reserve of infarct-related arties identifies reversible defects on noninvasive myocardial perfusion imaging early after myocardial infarction. J Am Coll Cardiol 2006;47:2187-93.

9. Beleslin et al. JNC current paper. 